\title{
Kinetic theory and dynamic structure factor of a condensate in the random phase approximation
}

\author{
Patrick Navez \\ Ecole Polytechnique, CP 165, Université Libre de Bruxelles, 1050 Brussels, Belgium
}

\begin{abstract}
We present the microscopic kinetic theory of a homogeneous dilute Bose condensed gas in the generalized random phase approximation (GRPA), which satisfies the following requirements: 1) the mass, momentum and energy conservation laws; 2) the H-theorem; 3) the superfluidity property and 4) the recovery of the Bogoliubov theory at zero temperature 11. In this approach, the condensate influences the binary collisional process between the two normal atoms, in the sense that their interaction force results from the mediation of a Bogoliubov collective excitation traveling throughout the condensate. Furthermore, as long as the Bose gas is stable, no collision happens between condensed and normal atoms. In this paper, we show how the kinetic theory in the GRPA allows to calculate the dynamic structure factor at finite temperature and when the normal and superfluid are in a relative motion. The obtained spectrum for this factor provides a prediction which, compared to the experimental results, allows to validate the GRPA.
\end{abstract}

PACS numbers:03.75.Hh, 03.75.Kk, 05.30.-d

\section{INTRODUCTION}

The kinetic theory of an interacting diluted Bose condensed gas remains still a challenging problem. The situation is indeed not entirely clear even though some microscopic description of the condensate dynamics have been attempted by many groups 2314. Some of the difficulties are really related to the basics of kinetics that Boltzmann developed more than one century ago, in order to derive an equation for the particle position and momentum distribution in a classical gas. This equation obeys the conservation laws for the mass, the momentum and the total energy density as well as the famous $\mathrm{H}$-theorem which allows to recover the second principle of thermodynamics. Strangely, the extension of that approach to a quantum Bose condensed gas 


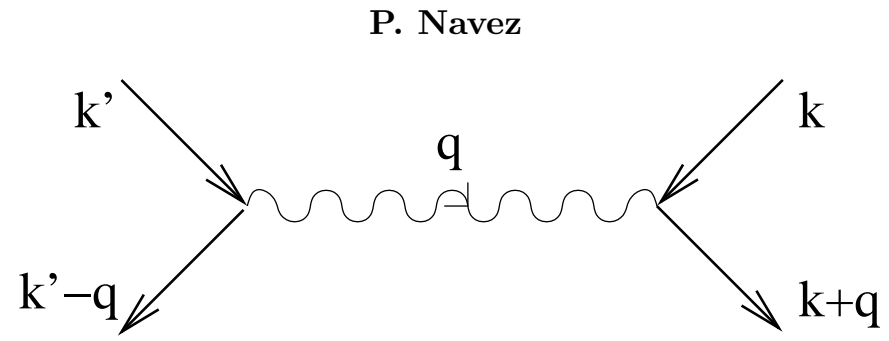

Fig. 1. Feymann diagram of the interaction of two normal atoms (full line) mediated by the Bogoliubov collective excitation (wavy line).

compatible with these conservation laws and the H-theorem has not seemed to attract a lot of attention. In addition, the kinetic equation must describe the superfluidity, in particular, the persistence of a non zero relative velocity between the normal and superfluid when it is below the critical velocity. Also, we expect to recover at zero temperature the Bogoliubov theory whose validity has been confirmed experimentally 5 . At first glance, all these requirements seem logical and well defined but an analysis of the literature reveals the difficulties to satisfy them in a unified theory. But, a solution to this problem has been recently presented with surprising properties 1 .

A kinetic equation has been derived by extending the generalized random phase approximation (GRPA), generally used for describing charged particles, to the case of a condensed atom gas. For a system of charged particles, the resulting equations describe the binary collision between the particles as a process of emission and absorption of a plasmon mediating the interaction. This plasmon corresponds to the longitudinal component of an electromagnetic wave in addition to the transverse ones. For neutral bosons, however, the condensate itself helps the thermal atoms to collide by transferring momentum and energy. The vehicle for this transmission is the Bogoliubov collective excitation energy with its dispersion relation characterizing a Goldstone boson (see Fig.1). Surprisingly also, provided that the condensate is stable, particle exchanges between the condensate and the thermal cloud are excluded since no collisions allow such a process. This point can be understood qualitatively by analogy with salt in the water. The interaction force between ions of salt is strongly attenuated due to the dielectric power of water to generate a counter polarized force. In the same way, due to its powerful "dielectric" behavior, the condensate can deform its wave function in a coherent way in order to diminish its potential energy and attenuate locally the potential interaction force displayed by any excited atom. In the GRPA, this effect is so strong that any atom in the condensate does not feel the presence of an excited atom anymore leading to the absence of any collision process. As a result, the particles of the normal and 


\section{Kinetic theory and dyn. struct. factor of a condensate in the RPA}

superfluid become invisible to each other and feel only the mean field force of the other fluid. This different picture allows to understand more simply why a relative velocity between the two fluids persists forever and leads to a metastable state characteristic of the phenomenon of superfluidity. This metastable state persists in time provided that the relative velocity does not exceed the sound velocity. Otherwise, the Landau criterion is not satisfied and the gas becomes unstable leading to some particle exchanges again.

The purpose of this paper is to show how to calculate the dynamic structure factor in the GRPA at finite temperature and in presence of a relative velocity. Since this factor can be measured in experiment, comparisons can serve to validate the GRPA approach. After recalling the basics of the GRPA method for the kinetics of a Bose gas in section 2, we show how to calculate this factor in section 3 .

\section{THE KINETIC EQUATION IN A HOMOGENEOUS GAS}

For any momentum $\mathbf{q}$, we define the density operator $\rho_{\mathbf{q}}=\sum_{\mathbf{k}} \rho_{\mathbf{k}, \mathbf{q}}$ where $\rho_{\mathbf{k}, \mathbf{q}}=c_{\mathbf{k}}^{\dagger} c_{\mathbf{k}+\mathbf{q}}$ and $c_{\mathbf{k}}\left(c_{\mathbf{k}}^{\dagger}\right)$ is the destruction (creation) operator. The GRPA assumes that the diagonal contribution is the dominant one i.e. $\left\langle\rho_{\mathbf{k}, \mathbf{q}}\right\rangle=\delta_{\mathbf{q}, \mathbf{0}} n_{\mathbf{k}}$. For $\mathbf{q}=0$, the time evolution in a volume $V$ of the population average of the atom with mass $m$ in the mode $\mathbf{k}$ is given by 1 ?

$$
i \frac{\partial}{\partial t} n_{\mathbf{k}}=\sum_{\mathbf{q}^{\prime}} \frac{2 \pi a}{m V}\left\langle\left(\rho_{\mathbf{k}, \mathbf{q}^{\prime}}-\rho_{\mathbf{k}-\mathbf{q}^{\prime}, \mathbf{q}^{\prime}}\right) \rho_{\mathbf{q}^{\prime}}^{\dagger}+\rho_{\mathbf{q}^{\prime}}^{\dagger}\left(\rho_{\mathbf{k}, \mathbf{q}^{\prime}}-\rho_{\mathbf{k}-\mathbf{q}^{\prime}, \mathbf{q}^{\prime}}\right)\right\rangle
$$

where $a$ is the scattering length. For $\mathbf{q} \neq 0$ in the GRPA, we neglect contribution that are quadratic in the non-diagonal operator in order to get an approximated linear equation:

$$
\begin{aligned}
& {\left[i \frac{\partial}{\partial t}+\epsilon_{\mathbf{k}}-\epsilon_{\mathbf{k}+\mathbf{q}}\right] \rho_{\mathbf{k}, \mathbf{q}}} \\
& \quad=\frac{4 \pi a}{m V} \sum_{\mathbf{k}^{\prime}}\left[n_{\mathbf{k}}\left(2-\delta_{\mathbf{k}, \mathbf{k}^{\prime}}-\delta_{\mathbf{k}-\mathbf{q}, \mathbf{k}^{\prime}}\right)-n_{\mathbf{k}+\mathbf{q}}\left(2-\delta_{\mathbf{k}, \mathbf{k}^{\prime}}-\delta_{\mathbf{k}+\mathbf{q}, \mathbf{k}^{\prime}}\right)\right] \rho_{\mathbf{k}^{\prime}, \mathbf{q}}
\end{aligned}
$$

where $\epsilon_{\mathbf{k}}=\mathbf{k}^{2} / 2 m$. This equation possesses two types of eigenvalues $\frac{6}{6}$. 1 ) the scattering solution with the spectrum given by the kinetic energy transfer: $\left.\omega=\epsilon_{\mathbf{k}+\mathbf{q}}-\epsilon_{\mathbf{k}} ; 2\right)$ for excitations involving the macroscopic condensate mode $n_{\mathbf{k}_{\mathbf{s}}}$ i.e. for $\mathbf{k}=\mathbf{k}_{\mathbf{s}}, \mathbf{k}_{\mathbf{s}}-\mathbf{q}$, the presence of interaction transforms the scattering solutions into collective solutions $\omega=\omega_{\mathbf{q}}-i \gamma_{\mathbf{q}}$. These are the zeroes of the dynamic dielectric function (DDF):

$$
\mathcal{K}(\mathbf{q}, \omega)=\mathcal{K}_{n}(\mathbf{q}, \omega)-\frac{\frac{8 \pi a n_{\mathbf{k}_{\mathbf{s}}}}{m V} \frac{\mathbf{q}^{2}}{m}}{\left(\omega+i 0_{+}-\frac{\mathbf{k}_{\mathbf{s}} \mathbf{q}}{m}\right)^{2}-\left(\frac{\mathbf{q}^{2}}{2 m}\right)^{2}+\frac{4 \pi a n_{\mathbf{k}_{\mathbf{s}}} \mathbf{q}^{2}}{m V}}
$$




\section{P. Navez}

where $\epsilon_{\mathbf{q}}^{B}=\sqrt{c^{2} \mathbf{q}^{2}+\left(\mathbf{q}^{2} / 2 m\right)^{2}}$ is the Bogoliubov excitation energy, $c=$ $\sqrt{4 \pi a n_{\mathbf{k}_{\mathbf{s}}} / m^{2} V}$ is the sound velocity and

$$
\mathcal{K}_{n}(\mathbf{q}, \omega)=1-\frac{8 \pi a}{m V} \sum_{\mathbf{k}} \frac{n_{\mathbf{k}}^{\prime}-n_{\mathbf{k}+\mathbf{q}}^{\prime}}{\omega+i 0_{+}-\frac{\mathbf{k} \cdot \mathbf{q}}{m}-\frac{\mathbf{q}^{2}}{2 m}}
$$

is the DDF of the normal fluid where $n_{\mathbf{k}}^{\prime}=\left(1-\delta_{\mathbf{k}, \mathbf{k}_{\mathbf{s}}}\right) n_{\mathbf{k}}$. The real part and imaginary part correspond to the Bogoliubov collective excitation and the Landau damping respectively. For a temperature close to zero, there are two solutions ${ }^{\mathbb{1}}: \omega_{\mathbf{q}}^{ \pm} \simeq \frac{\mathbf{k}_{\mathbf{s}} \mathbf{q}}{m} \pm \epsilon_{\mathbf{q}}^{B}, \gamma_{\mathbf{q}}^{ \pm} \simeq \pm \operatorname{Im} \mathcal{K}_{n}\left(\mathbf{q}, \omega_{\mathbf{q}}^{ \pm}\right) c^{2} \mathbf{q}^{2} / \epsilon_{\mathbf{q}}^{B}$.

The substitution of the solution of Eq.(2) into (11) allows finally to get the GRPA kinetic equation for a Bose gas 1 . As explained in the introduction we find that $\partial n_{\mathbf{k}_{\mathbf{s}}} / \partial t=0$ i.e. the condensed particle number is a constant of motion and thus no particle is exchanged with the normal fluid. For the normal fluid, the populations in each mode obey the balance equations:

$$
\begin{array}{r}
\frac{\partial}{\partial t} n_{\mathbf{k}}^{\prime}=\sum_{\mathbf{q}, \mathbf{k}^{\prime}}\left|\frac{\frac{8 \pi a}{m V}}{\mathcal{K}\left(\mathbf{q}, \epsilon_{\mathbf{k}+\mathbf{q}}-\epsilon_{\mathbf{k}}\right)}\right|^{2} \pi \delta\left(\epsilon_{\mathbf{k}+\mathbf{q}}+\epsilon_{\mathbf{k}^{\prime}-\mathbf{q}}-\epsilon_{\mathbf{k}}-\epsilon_{\mathbf{k}^{\prime}}\right) \\
{\left[n_{\mathbf{k}+\mathbf{q}}^{\prime} n_{\mathbf{k}^{\prime}-\mathbf{q}}^{\prime}\left(n_{\mathbf{k}}^{\prime}+1\right)\left(n_{\mathbf{k}^{\prime}}^{\prime}+1\right)-n_{\mathbf{k}}^{\prime} n_{\mathbf{k}^{\prime}}^{\prime}\left(n_{\mathbf{k}+\mathbf{q}}^{\prime}+1\right)\left(n_{\mathbf{k}^{\prime}-\mathbf{q}}^{\prime}+1\right)\right]}
\end{array}
$$

These show that two normal particles collide through emission and absorption of a Bogoliubov collective excitation, called "condenson" (see Fig.1). The dispersion relation for an excitation of momentum $\mathbf{q}$ is given by the pole of the DDF which plays the role of a propagator in (5). For a momentum $\mathbf{q}$, this excitation has the energy $\omega_{\mathbf{q}}$ and a decay rate, due to its absorption by a thermal atom, given by the Landau damping $\gamma_{\mathbf{q}}$. Similarly to the plasma $\operatorname{case}^{7}$, these equations are valid provided that the collective oscillations are damped i.e. $\gamma_{\mathbf{q}} \geq 0$. Otherwise, they are amplified exponentially and the Bose gas becomes unstable. The case with instability leads to different kinetic equations and is not treated here. But the instable regime allows particles exchange between the two fluids. The equilibrium solution of (5) for a normal fluid at rest is given by $n_{\mathbf{k}}^{\prime e q}=1 / \exp \left[\beta\left(\epsilon_{\mathbf{k}}-\mu\right)\right]-1$. In this case when the temperature is close to zero, we find that:

$$
\operatorname{Im} \mathcal{K}_{n}(\mathbf{q}, \omega)=\frac{2 a m}{\beta|\mathbf{q}|} \ln \left(\frac{1-\exp \left\{-\beta\left[m \frac{\left(\omega+\mathbf{q}^{2} / 2 m\right)^{2}}{2 \mathbf{q}^{2}}-\mu\right]\right\}}{1-\exp \left\{-\beta\left[m \frac{\left(\omega-\mathbf{q}^{2} / 2 m\right)^{2}}{2 \mathbf{q}^{2}}-\mu\right]\right\}}\right)
$$

which is a positive function for $\omega=\omega_{\mathbf{q}}^{ \pm} \geq 0$ and negative otherwise. Thus, we deduce that the stability condition $\gamma_{\mathbf{q}}^{ \pm} \geq 0$ is equivalent to the Landau criterion $\epsilon_{\mathbf{q}}^{B} \geq \mathbf{k}_{\mathbf{s}} \cdot \mathbf{q} / m$ i.e. $\left|\mathbf{k}_{\mathbf{s}} / m\right| \leq c$. In other words, the condensate is stable if its velocity relative to the normal fluid is smaller than the sound velocity. 


\section{Kinetic theory and dyn. struct. factor of a condensate in the RPA}

\section{THE DYNAMIC STRUCTURE FACTOR}

The dynamic structure factor is defined as:

$$
S(\mathbf{q}, \omega)=\lim _{\tau \rightarrow \infty} \int_{V} d \mathbf{r} \int_{-\infty}^{\infty} \frac{d \tau^{\prime}}{2 \pi} e^{-i\left(\mathbf{q} \cdot \mathbf{r}-\omega \tau^{\prime}\right)}\left\langle\rho_{\mathbf{q}}(\tau) \rho_{-\mathbf{q}}\left(\tau+\tau^{\prime}\right)\right\rangle
$$

This function is calculated within the GRPA and within the assumptions that the normal fluid is in thermal equilibrium at rest. We start with the initial condition that for $\tau=0$ the correlation function contains only non interacting contribution 1 : $\left.\left\langle\rho_{\mathbf{k}^{\prime},-\mathbf{q}} \rho_{\mathbf{k}, \mathbf{q}}\right\rangle\right|_{\tau=0}=\left(n_{\mathbf{k}}+1\right) n_{\mathbf{k}^{\prime}} \delta_{\mathbf{k}^{\prime}-\mathbf{k}, \mathbf{q}}\left(1-\delta_{\mathbf{q}, 0}\right)+$ $n_{\mathbf{k}} n_{\mathbf{k}^{\prime}} \delta_{\mathbf{q}, 0}$. Through Eq.(5), the evolution of the system will create for $\tau \rightarrow \infty$ some correlation due to the presence of the interaction between particles. Similarly to the plasma case, the resulting dynamic structure factor is proportional to the inverse of the DDF 7 :

$$
S(\mathbf{q}, \omega)=\delta(\omega) \delta_{\mathbf{q}, \mathbf{0}} N^{2}+\frac{1}{2 \pi} \frac{1}{\exp (-\beta \omega)-1} \frac{m V}{4 \pi a} \operatorname{Im}\left(\frac{1}{\mathcal{K}(\mathbf{q}, \omega)}\right)\left(1-\delta_{\mathbf{q}, \mathbf{0}}\right)
$$

The relevant contributions are near the poles of $\mathcal{K}(\mathbf{q}, \omega)$. At temperature close to zero, we obtain the approximated expression:

$$
\frac{1}{\mathcal{K}(\mathbf{q}, \omega)}=\frac{c^{2} \mathbf{q}^{2}}{\epsilon_{\mathbf{q}}^{B}}\left(\frac{1}{\omega-\omega_{\mathbf{q}}^{+}+i \gamma_{\mathbf{q}}^{+}}+\frac{1}{\omega-\omega_{\mathbf{q}}^{-}+i \gamma_{\mathbf{q}}^{-}}\right)
$$

Eq.(8) and (92) are the main results of this paper and generalize previous results $\sqrt{518}$ for any temperature in the presence of a relative velocity between the two fluids. Due to the asymmetric dispersion relation, this factor has

two asymmetric peaks with usual intensity given by $S(\mathbf{q})=\mathbf{q}^{2} / 2 m \epsilon_{\mathbf{q}}^{B}$. Also the presence of temperature gives rise to the broadening of the peaks with a width corresponding to the Landau damping. When the relative velocity approaches the sound velocity, the asymmetric Bogoliubov frequency approaches zero for some non zero momentum for which the Landau damping gets closer to zero. Beyond that velocity, in some regions of the momentum, both the resonance frequency and the Landau damping become negative. In that case, the gas becomes instable leading to non equilibrium dynamics. Thus the experimental analysis of the dynamic structure factor provides a different way to confirm that the sound velocity is the critical velocity beyond which some instability should appear. In real experiments, the width comes mainly from inhomogeneous broadening but, in principle, the asymmetry between the peaks must be observed. For this purpose, we need to create a situation with a non zero relative velocity ${ }^{9}$. Knowing the spatial density and velocity distribution of the condensate, we can use the local 


\section{P. Navez}

density approximation to calculate the line shape of the Bragg excitations and test the validity of the results 58 .

To conclude, the GRPA formalism confirms previous results established for the dynamic structure factor. Moreover, it allows to calculate this quantity from (7) not only for an equilibrium distribution function but also for a non equilibrium one. Of special interest is the metastable state of superfluidity from which an explicit expression has been obtained for temperature close to zero and which displays the transition from metastability to instability. This transition is dictated by the Landau criterion which delimits the region in which the dynamic structure factor is well defined. Beyond that region this function is not stationary anymore due to unstable dynamics.

\section{ACKNOWLEDGMENTS}

PN thanks W. Ketterle, J. Tempere and J. Devreese for discussions. PN acknowledges financial support from the Communauté Française de Belgique under grant ARC 00/05-251, from the IUAP programme of the Belgian government under grant $\mathrm{V}-18$, and from the EU under project RESQ (IST2001-35759).

\section{REFERENCES}

1. P. Navez, cond-mat/0309319

2. A. J. Leggett, Rev. Mod. Phys. 73, 307 (2001).

3. E. Zaremba, T. Nikuni, and A. Griffin, J. Low Temp. Phys. 116, 227 (1999).

4. R. Walser, J. Williams, J. Cooper, M. Holland, Phys. Rev. A, 59, 3878 (1999).

5. D.M. Stamper-Kurn, A.P. Chikatur, A. Görlitz, S. Inouye, S. Gupta, D.E. Pritchard, and W. Ketterle, Phys. Rev. Lett. 83, 2876 (1999).

6. D. Pines and P. Nozières, The theory of Quantum Liquids, vol 1 (Benjamin, New York,1966).

7. S.Ichimaru, Frontier in Physics: Statistical Plasma Physics, Vol I: Basic Principle (Addison Wesley, 1992).

8. F. Zambelli, L. Pitaevskii, D.M. Stamper-Kurn and S. Stringari, Phys. Rev. A 61, 063608 (2000).

9. D.M. Stamper-Kurn, H.-J. Miesner, S. Inouye, M.R. Andrews, and W. Ketterle, Phys. Rev. Lett. 81, 500 (1998). 